\title{
Quality appraisal in systematic reviews of public health interventions: an empirical study on the impact of choice of tool on meta-analysis
}

\author{
Peer H Voss, Eva A Rehfuess
}

Institute for Medical Informatics, Biometry and Epidemiology, University of Munich, Munich, Germany

\section{Correspondence to}

Dr Eva Rehfuess, Institute for Medical Informatics, Biometry and Epidemiology, University of Munich, Marchioninistrasse 15 81377 Munich, Germany; rehfuess@ibe.med. uni-muenchen.de

Accepted 8 May 2012 Published Online First 31 July 2012

\begin{abstract}
Introduction Systematic reviews are a cornerstone of evidence-based public health. The method of appraising the quality of different intervention and observational study designs in such reviews remains an important challenge. This article examines the applicability of selected quality appraisal tools (OATs) and the impact of choice of tool on the meta-analysis of a published systematic review.

Methods The authors selected a systematic review on the effectiveness of hand washing with soap in preventing diarrhoea, covering a range of epidemiological study designs. 6 OATs were used to assess 13 studies meeting their inclusion criteria; component sections/questions were coded numerically to derive a summary score between -1 (low quality) and +1 (high quality) for each OAT and study. Heterogeneity in study quality was evaluated graphically using traffic light schemes and spider charts. Random effects meta-analysis was undertaken for all studies; sensitivity analyses for each OAT included only those studies with a score of 0 or above.
\end{abstract}

Results The authors found substantial heterogeneity in summary scores for a given study. Their main metaanalysis yielded an OR of $0.60(95 \% \mathrm{Cl} 0.47$ to 0.77$)$ with most sensitivity analyses giving similar pooled effect sizes with wider Cls.

Discussion The six OATs differ greatly in applicability across study designs, approach to quality appraisal (ie, scale vs checklist, presence/absence of summary score), coverage of domains and quality of component questions and answers. Learning from advantages and disadvantages of each QAT, we recommend research into the development of a reliable OAT with a broad applicability across study designs.

\section{INTRODUCTION}

The concept of evidence-based public health is receiving increasing attention. One proposed definition describes evidence-based public health as 'the development, implementation and evaluation of effective programmes and policies in public health through application of principles of scientific reasoning, including systematic uses of data and information systems and appropriate use of behavioural science theory and programme planning models'. In this context, assessing the effectiveness of public health interventions in a reliable way is critical, and systematic reviews are becoming more and more important. ${ }^{2}$ There is much discussion about the challenges that arise when this method of evidence synthesis, originally developed for evidencebased medicine, is transferred to public health.
In particular, limiting systematic reviews of public health interventions to randomised controlled trials (RCTs) may dismiss as noise much of what others would consider to be the signal. ${ }^{3}$ Therefore, one key question is 'how low one should go' in the hierarchy of study designs. ${ }^{4}$ Beyond determining whether any evidence is found at all in situations where RCTs are not available, the decision on which study designs to include involves a difficult trade-off between maximising internal validity and producing reliable pooled effect estimates versus ensuring that findings are transferable to real-life settings and of policy relevance.

A related challenge is how to assess study quality when a variety of study designs comprising, for example, standard observational study designs, individually and cluster-randomised trials, as well as non-randomised intervention trials, are considered. Using study design alone as the guiding principle may mix well-conducted studies with high internal validity with poorly-conducted studies with low internal validity. Careful and comprehensive quality appraisal is therefore critical in ensuring that studies with a high risk of bias are not allowed to contribute to pooled effect estimates. While quality appraisal of randomised studies tends to follow established procedures, there is lack of agreement on how to assess the quality of non-randomised studies. Quality appraisal tools (OATs) show much variability in development process, intent, construction and psychometric properties $^{5-8}$ and are distinct with respect to the aspects of study quality they cover and the weight assigned to them. These differences in approach may lead to discrepant conclusions on which studies provide internally valid results and which do not, and it can thus be suspected that the decision to use one OAT versus another influences the decision on which studies are allowed to contribute to meta-analysis and, consequently, the size and CI of the pooled effect estimate.

This article examines the applicability of selected OATs to different study designs, explores how the choice of tool impacts the meta-analysis of a published systematic review of a public health intervention and makes recommendations for quality appraisal in systematic reviews of public health interventions.

\section{METHODS \\ Selection of systematic review}

We attempted to identify a published systematic review that (1) examines a relatively simple public health intervention, (2) includes a range of 
randomised and non-randomised study designs, (3) comprises fewer than 25 primary studies (for reasons of feasibility) and (4) conducts meta-analysis. A systematic review of the effectiveness of hand washing with soap in preventing diarrhoea by Curtis and Cairncross met all of these criteria. ${ }^{9}$

As this review included only studies up to the year 2001, we searched PubMed and contacted researchers involved with the comparative risk assessment for water, sanitation and hygiene as part of the Global Burden of Disease 2010 study. ${ }^{10}$ This yielded five related systematic reviews ${ }^{11-15}$ as well as an incomplete update of the originally identified review; ${ }^{16}$ these are summarised in our systematic review protocol (available upon request). As none of these more recent reviews had searched the literature for observational studies and as the objective of our study was not to provide the most up-to-date estimate of the effectiveness of hand washing but to explore how the choice of OAT influences an estimate obtained through combining a variety of epidemiological study designs, we used the original systematic review. ${ }^{9}$

\section{Selection of OATs}

Six OATs were purposively selected for testing based on their previously demonstrated quality and/or their particular relevance to systematic reviews of public health interventions. Deeks and colleagues assessed 194 OATs; 14 covered at least five internal validity domains and three core items for nonrandomised studies and were thus considered most reliable. ${ }^{5}$ Among these, we selected the Effective Public Health Practice Project tool (EPHPPT; recommended by the Cochrane Public Health Group), ${ }^{17}$ the Critical Appraisal Skills Programme (CASP) tool ${ }^{18}$ and the Newcastle-Ottawa Scale (NOS) for their frequent use in the field of public health. ${ }^{19}$ Three additional, more recently developed OATs were also tested. Based on the NOS, a group of researchers at the University of Liverpool designed checklists for four study designs and used these in several systematic reviews as part of the Global Burden of Disease 2010 study's comparative assessment of health risks; ${ }^{10}$ selected components of the Liverpool tool (ie, exposure and outcome assessment) are adapted to the given research question, ${ }^{20}$ in our case based on requirements for reliable and valid assessment of hand washing practices and diarrhoea. Finally, the Graphical Appraisal Tool for Epidemiological Studies (GATE) ${ }^{21}$ is employed by the Public Health Excellence Centre of the United Kingdom National Institute for Health and Clinical Excellence, and the Cochrane Collaboration Risk of Bias tool (ROB) represents an accepted standard for systematic reviews. The latter is currently being modified for non-randomised studies; we tested the version presented at an international workshop in Munich in
November $2010^{22}$ but employed a three-point (ie, low, moderate, high) rather than five-point rating for risk of bias.

\section{Study inclusion criteria}

We adopted the inclusion criteria by Curtis and Cairncross: ${ }^{9}$ (1) hand washing with soap as the intervention (excluding combined interventions and interventions promoting hand washing without soap), (2) diarrhoeal diseases as the health outcome and (3) no age restrictions, but modified these to consider only studies conducted (4) in developing countries and (5) community settings. We made these restrictions as deriving effect estimates across very distinct implementation settings (ie, childcare or other institutional setting vs home setting) and national contexts (ie, developing vs industrialised country) would not be meaningful.

\section{Data extraction and quality appraisal}

For all included studies, data extraction was undertaken independently by both authors using a modified version of the Cochrane Collaboration data extraction form. ${ }^{23}$ Subsequently, both authors independently applied all six OATs, compared their results and resolved any discrepancies in quality ratings through discussion.

\section{Evaluation of QATs}

Each OAT was evaluated regarding its applicability to different study designs, coverage of components, and quality of component questions and answers. To be able to make a OAT-informed decision on whether a given study should be included in metaanalysis or not, we had to follow an objective, transparent process, rather than letting our overall understanding of the weaknesses of a given study, developed through assessment with all six OATs, drive that decision. While three OATs generate numerical or graded summary scores, which could be used as a basis for an inclusion/exclusion decision, the other three are checklists and do not offer any such guidance. Therefore, to enable meaningful comparisons across all six OATs, we coded individual components of each tool on a scale ranging from -1 (low quality) to 0 (moderate quality) and +1 (high quality). In the case of GATE, for example, individual component ratings of '-', 'not reported/not applicable/+' and ' ++ ' for each question were coded as $-1,0$ and +1 , respectively. Ultimately, for each study and OAT, a summary score ranging from -1 to +1 was derived as the sum of individual component ratings divided by the number of components. We graphically depicted homogeneity versus heterogeneity in quality appraisal with different OATs by adapting the Cochrane traffic light scheme and developing spider charts (see figure 1 for explanation).
Figure 1 (A) Traffic light scheme. (B) Spider chart. The traffic light scheme provides a rapid graphical overview of whether a study scores high $(+)$, moderate (?) or low (-) on different quality domains/questions; similarity in predominant signs (i.e.,+ ?, - ) indicates good agreement between different QATs. The spider chart approximates a circle when different QATs result in a similar quality assessment, with a larger circle indicating a higher-quality study and a smaller circle indicating a lower-quality study. For Hussein Gasem et $a^{27}$ large differences in predominant signs and a spider chart with kinks suggests that assessment of study quality varies between QATs. CASP, Critical Appraisal Skills Programme; EPHPPT, Effective Public Health Practice

suggests that assessment of study quality varies between OATs. CASP, Critical Appraisal Skills Programme; EPHPPT, Effective Public Health Practice
Project tool; GATE, Graphical Appraisal Tool for Epidemiological Studies; NOS, Newcastle-Ottawa Scale; QAT, quality appraisal tool; ROB, Cochrane Collaboration Risk of Bias tool.
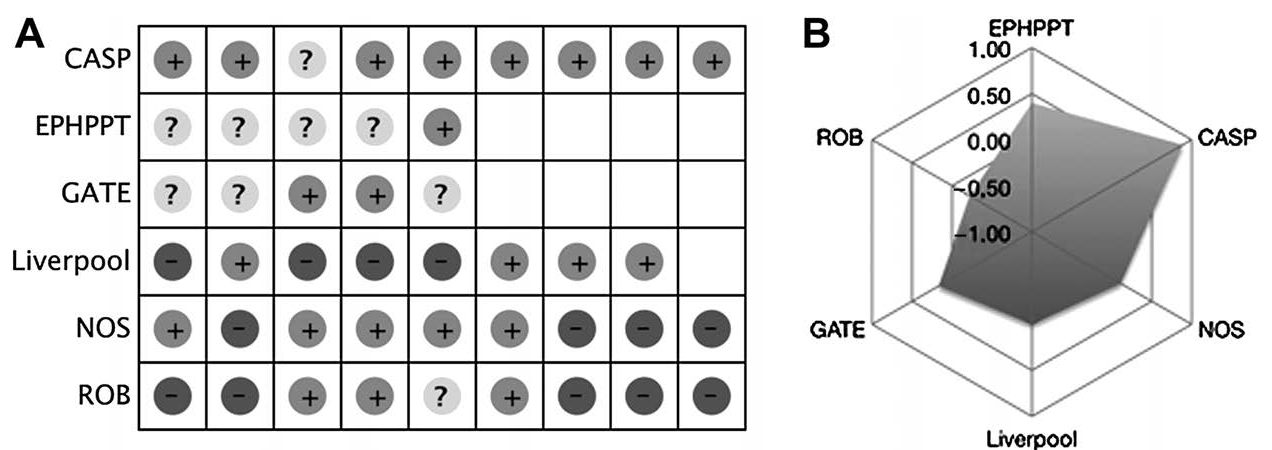


\section{Meta-analyses}

The approach to meta-analysis was first to pool all eligible studies and then to carry out sensitivity analyses to assess the impact of OAT choice. Studies eligible for meta-analysis provided an estimate of effect with 95\% CI (or the information to calculate these); we attempted to obtain missing information by approaching the first and senior authors of studies. Effect estimates for studies with different measures of hand washing (eg, before eating, after defecation) or different diarrhoeal disease outcomes (eg, dysentery, shigellosis) were combined by averaging, if they concerned the same sample group. If they concerned different sample groups, they were reported separately. Effect estimates were included as ORs; where available adjusted ORs were used.

We undertook one sensitivity analysis for each OAT, including only studies with a coding of 0 or above, and additional sensitivity analyses for EPHPPT (including only studies with an overall rating of moderate or high) and GATE (including only studies scoring + or ++ on the overall assessment of internal and external validity). We conducted random effects meta-analyses using the generic inverse variance method; all analyses were run in Review Manager V.5.1.2 (Copenhagen, Denmark).

\section{RESULTS}

Table 1 gives an overview of the characteristics of the six OATs, classifying these as scales (ie, resulting in a numerical summary score) and checklists (ie, a list of items with or without a qualitative summary score). OATs differ in terms of study designs considered and number of components included. Most importantly, there is much variation in the full or partial coverage of key study quality domains as defined by Sanderson et al. ${ }^{7}$ Confounding is the only key domain addressed by all OATs; conflict of interest is not considered by any tool.

Thirteen studies met our inclusion criteria ${ }^{24-36}$ (table 2), comprising one RCT, four non-randomised intervention trials, one cohort study, six case-control studies and one cross-sectional study, and covering Africa (three studies), Asia (nine studies) and Latin America (one study). The summary scores in table 2 reveal major shortcomings in most studies (eg, inadequate adjustment for confounding, poor control selection). They also highlight substantial heterogeneity in results, as graphically confirmed by traffic light schemes (figure 1a) and spider charts (figure 1b) for each study.

Meta-analysis was conducted using 13 estimates from 12 included studies; ORs and 95\% CIs were neither available nor calculable for Yeager and colleagues. ${ }^{36}$ Figure 2 shows the ORs for all included studies yielding a pooled OR of 0.60 (95\% CI 0.47 to 0.77$)$. An asymmetric funnel plot suggests high risk of publication bias.

The results of the sensitivity analyses are shown in table 3 . First, there are large differences in the number of studies included. With nine out of 13 risk estimates included, CASP appears most inclusive in relation to study quality; due to negative ratings for all studies, the $\mathrm{ROB}$ does not allow a pooled risk estimate to be derived. Using an alternative summary score results in the inclusion of an additional study for the EPHPPT and no change for GATE. Second, three studies ${ }^{28} 3235$ are not included in any sensitivity analyses, which may imply that all six OATs are able to distinguish between very poor quality studies and moderate to high quality studies. Finally, pooled effect estimates are remarkably consistent across sensitivity analyses although there is considerable variation in the precision of the estimate; except for the NOS, all sensitivity analyses yield statistically significant pooled ORs.

\section{DISCUSSION}

\section{Key findings}

We applied selected OATs to a systematic review of the effectiveness of hand washing in preventing diarrhoea. These analyses indicate substantial differences between OATs with respect to their applicability across study designs and the relative importance assigned to different quality domains, and show large differences in the overall score for a given study. As the same pair of researchers undertook data extraction and quality appraisal for all studies and OATs, it is likely that this heterogeneity indicates real differences in the way OATs assess the quality of a study.

Our main meta-analysis based on 13 estimates from 12 studies yielded a pooled OR of 0.60 (95\% CI 0.47 to 0.77 ). This finding is roughly equivalent to the pooled risk estimate of 1.74 (95\% CI 1.39 to 2.18 ) in the meta-analysis of Curtis and Cairncross including 17 studies. $^{9}$ Even though the number of included studies varies widely, the pooled ORs in our sensitivity analyses are remarkably robust, although implications of the observed differences for decision-making become more apparent when ORs are converted to numbers needed to treat (NNT). For example, with an assumed control risk of 0.3 , the smallest pooled OR of 0.57 (GATE) translates into an NNT of 10 compared with an NNT of 14 for the largest pooled OR of 0.69 (NOS) (table 3). It is noteworthy that more recently conducted systematic reviews of the effectiveness of hand washing, which due to their focus on intervention studies presumably include more higher quality studies, find slightly lower average risk reductions of $36 \%,{ }^{11} 32 \%,{ }^{12} 25 \%-37 \%{ }^{13}$ and $31 \%$. ${ }^{15}$ This suggests that systematic reviews that include non-randomised studies may overestimate the effectiveness of a given intervention; including all intervention and observational studies independent of their quality appears to lead to a false precision in the pooled effect estimate.

\section{Study limitations}

Thirteen studies constitute a small sample size, in particular with only one cohort study and none of the more sophisticated study designs (eg, interrupted time-series studies) included. Our sample is relatively old (dating from 1982 to 2001), which partly explains why most studies are of rather poor quality. Epidemiological practice and reporting standards have evolved since, and it is likely that a more recently conducted systematic review including studies following TREND, ${ }^{37}$ CONSORT $^{38}$ or STROBE $^{39}$ reporting requirements would yield a higher quality sample.

Quality appraisal is always somewhat subjective ${ }^{40}$ and the judgement about how well a particular study meets a specific criterion will vary when different groups of researchers are involved. We have tried to be as transparent as possible in our rating of studies, quantitative coding and decisions about including/excluding studies in sensitivity analyses. As a basis for being able to conduct OAT-informed meta-analyses across tools, we assigned numerical values to each answer category in a given OAT, which may have skewed the scale intended by the developers.

We assumed equal weighting of components or questions in deriving a quantitative summary score for each OAT. We are aware of the discussions on the benefits and drawbacks of deriving a summary score and realise that our approach may be against the intention of the developers of some tools as it does not distinguish appropriately between components that are critical to the overall validity of a study and those of lesser importance. This may, for example, lead to a false reassurance by allowing a study with one serious flaw but an overall moderate 


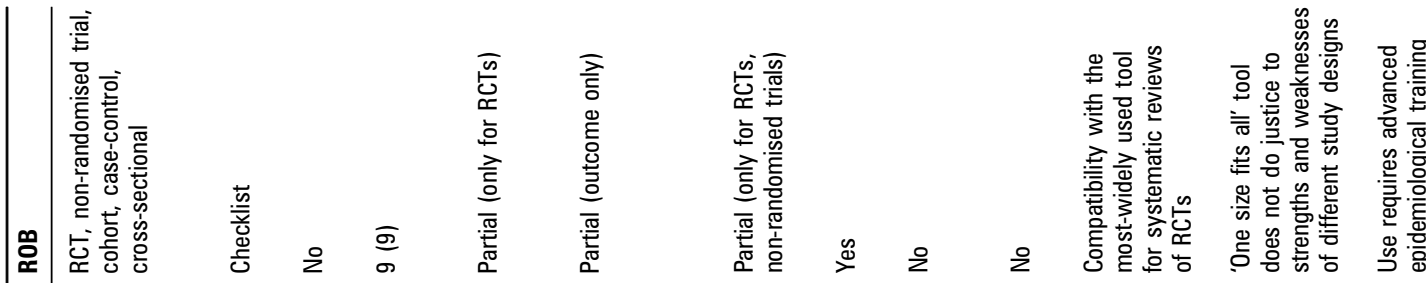

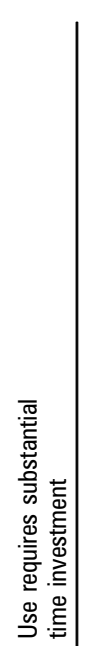

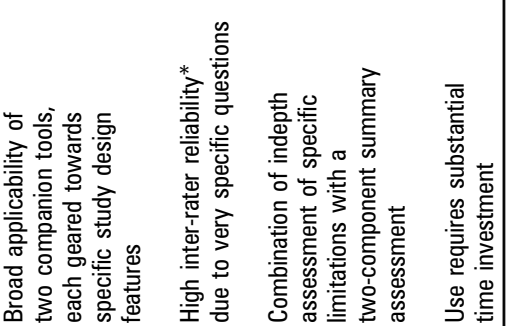

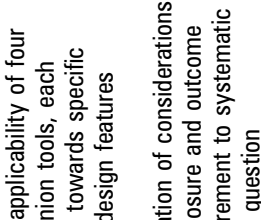

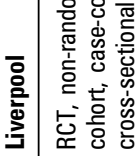

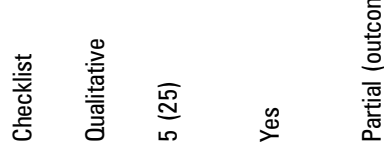<smiles>C[C@H]1[C@@H]2C[C@@H](C)[C@H]1C2</smiles>
$\stackrel{\mathscr{\infty}}{\rightleftharpoons}$

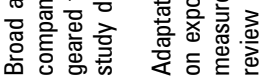<smiles>C[AlH]</smiles>

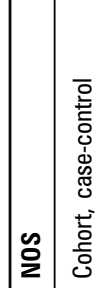
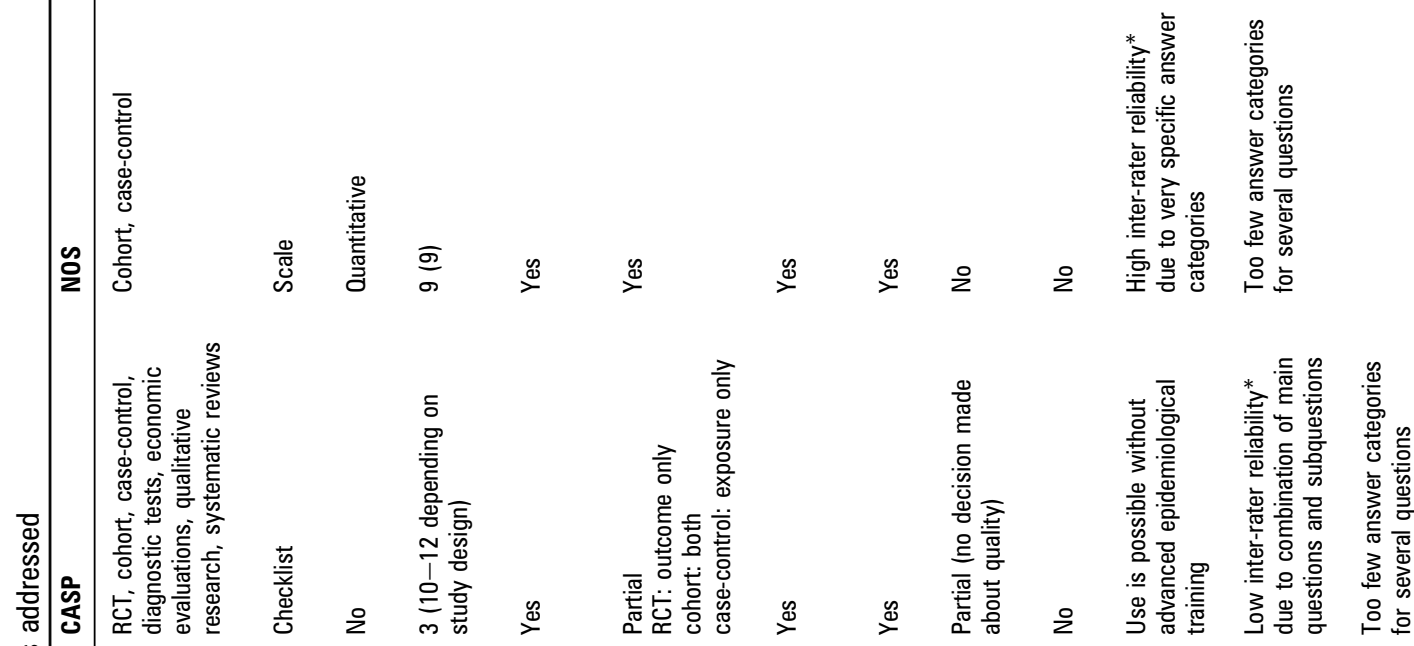
Table 2 Summary scores* of studies based on different QATs

\begin{tabular}{|c|c|c|c|c|c|c|c|c|}
\hline ID & Author(s) (year) & Study design & EPHPPT & CASP & NOS & Liverpool & GATE & ROB \\
\hline $1 \mathrm{a}$ & Birmingham et al (1997) & Case-control study & -0.40 & 0.44 & -0.11 & -0.42 & -0.39 & -0.56 \\
\hline $1 b$ & Birmingham et al (1997) & Case-control study & -0.40 & 0.33 & 0.11 & -0.38 & -0.33 & -0.56 \\
\hline 2 & Han and Hlaing (1989) & RCT & 0.50 & 0.88 & NA & 0.50 & 0.24 & -0.33 \\
\hline 3 & Hoque et al (1999) & Case-control study & 0.40 & 0.56 & 0.33 & 0.50 & 0.39 & -0.22 \\
\hline 4 & Hussein Gasem et al (2001) & Case-control study & 0.40 & 0.89 & 0.11 & 0.00 & 0.17 & -0.22 \\
\hline 5 & Khan (1982) & Non-randomised trial & -0.83 & NA & NA & -0.11 & -0.48 & -1.00 \\
\hline 6 & Khin et al (1994) & Case-control study & -0.60 & 0.00 & -0.11 & -0.50 & -0.61 & -0.67 \\
\hline 7 & Peterson et al (1998) & Cohort study & -0.17 & 0.50 & 0.33 & -0.67 & -0.17 & -0.78 \\
\hline 8 & Shahid et al (1996) & Non-randomised trial & -0.17 & NA & NA & -0.33 & 0.16 & -0.44 \\
\hline 9 & Sircar et al (1987) & Non-randomised trial & -0.67 & NA & NA & -0.50 & -0.36 & -0.89 \\
\hline 10 & St Louis et al (1990) & Case-control study & -0.40 & 0.22 & -0.11 & -0.56 & 0.11 & -0.67 \\
\hline 11 & Velema et al (1997) & Case-control study & -0.40 & 0.00 & 0.11 & -0.50 & -0.06 & -0.44 \\
\hline 12 & Wilson et al (1991) & Non-randomised trial & -0.67 & NA & NA & -0.83 & -0.20 & -0.89 \\
\hline 13 & Yeager et al (1991) & Household survey & 0.00 & NA & NA & -0.44 & -0.06 & -0.33 \\
\hline
\end{tabular}

*Summary scores can range from -1 (low quality) to 0 (moderate quality) and +1 (high quality).

CASP, Critical Appraisal Skills Programme; EPHPPT, Effective Public Health Practice Project tool; GATE, Graphical Appraisal Tool for Epidemiological Studies; NA, not applicable; NOS,

Newcastle-Ottawa Scale; QAT, quality appraisal tool; RCT, randomised controlled trial; ROB, Cochrane Collaboration Risk of Bias tool.

rating to contribute to a pooled analysis. We believe that summary scores are useful in pinpointing to potentially problematic studies but should not be used at the expense of a detailed investigation and documentation of specific study limitations. In addition to using such scores to decide on inclusion/exclusion of studies in meta-analysis or to inform sensitivity analyses, variation in selected critical quality criteria, such as selection bias or confounding, could equally be used to guide sensitivity analyses. Similarly, the decision to include all studies scoring 0 or above is somewhat arbitrary. It would be informative to explore how different ways of deriving a summary score and more or less rigorous cut-off values might influence the results.

Despite these limitations, we believe that this first attempt to systematically apply several OATs across all studies within a systematic review of a public health intervention provides relevant insights into the importance of quality appraisal and the impact of choice of OAT on meta-analysis.

\section{Evaluation of QATs}

In terms of applicability across study designs, EPHPPT and ROB follow the 'level playing field' approach, that is, all study designs compete at the same level. NOS and Liverpool, on the other hand, develop a set of OATs for specific study designs, suggesting that each study should be planned, conducted and analysed as well as possible given inherent design constraints.

The pursuit of the scale versus checklist approach has important implications for systematic reviews. Summary scores (eg, EPHPPT, NOS, Liverpool) enable a simple and semiquantitative decision about whether or not a study should be included in meta-analysis. Checklists (eg, ROB, CASP) make such a decision more difficult but could nevertheless be used to inform sensitivity analyses (eg, exclusion of studies with a certain number of weak ratings). GATE combines the two approaches by guiding the evaluator through a list of items and then asking him or her to draw a conclusion about internal and external validity.

OATs clearly differ in the relative importance assigned to different research characteristics, partly due to their development history ${ }^{40}$ (table 1). For example, the ROB was originally developed for RCTs and subsequently modified, which explains why several critical considerations for non-randomised studies (eg, exposure measurement) are not addressed and why most non-randomised studies automatically score low on key components (eg, sequence generation, allocation concealment). It is also noteworthy that some components (eg, statistical analysis in EPHPPT) are assessed but not rated.

\section{Quality of component questions}

The degree to which a question is formulated in a specific (eg, GATE's distinction among source population, eligible population and selected participants) or less specific way (eg, Liverpool's general enquiry about selection bias) has an impact on the subjectivity of the answer. While we did not formally assess inter-rater reliability (see also table 1), we noted that GATE and NOS resulted in fewer discrepancies than the other OATs. Likewise, we observed that the combination of main questions with subquestions (eg, CASP) increases variability in answers. Most OATs do not foresee adaptation to the systematic review question. We found, however, that the definition of a gold
Figure 2 Meta-analysis for the effect of hand washing with soap in diarrhoeal disease.

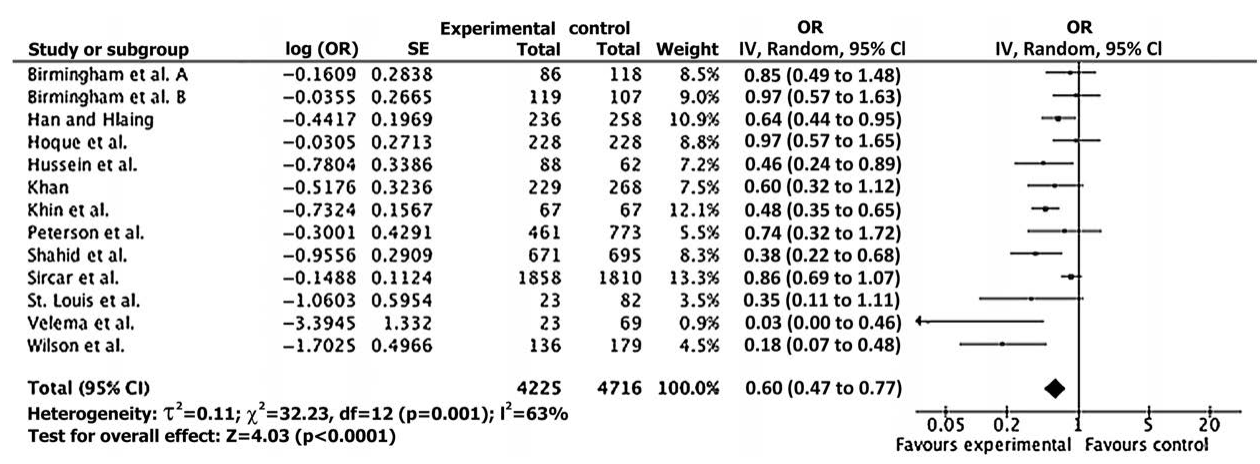


Table 3 Sensitivity analyses for the effect of hand washing with soap on diarrhoeal disease according to different quality appraisal tools

\begin{tabular}{llll}
\hline & $\begin{array}{l}\text { Pooled OR } \\
\text { (95\% CI) }\end{array}$ & $\begin{array}{l}\text { \% Change in } \\
\text { pooled OR }\end{array}$ & Included studies $\S$ \\
\hline Main meta-analysis & $0.60(0.47$ to 0.77$)$ & - & $1-12$ \\
EPHPPT* & $0.68(0.47$ to 0.98$)$ & +13.33 & $2,3,4$ \\
EPHPPT $\dagger$ & $0.59(0.41$ to 0.86$)$ & -1.67 & $2,3,4,8$ \\
CASP & $0.64(0.49$ to 0.85$)$ & +6.67 & $1 \mathrm{a}, \mathrm{b}, 2,3,4$, \\
& & & $6,7,10,11$ \\
NOS & $0.69(0.42$ to 1.14$)$ & +15.00 & $1 \mathrm{~b}, 3,4,7,11$ \\
Liverpool & $0.68(0.47$ to 0.98$)$ & +13.33 & $2,3,4$ \\
GATE* & $0.57(0.40$ to 0.81$)$ & -0.05 & $2,3,4,8,10$ \\
GATE $\ddagger$ & $0.57(0.40$ to 0.81$)$ & -0.05 & $2,3,4,8,10$ \\
ROB & No study included & No study & No study included \\
& & included & \\
\hline
\end{tabular}

*Inclusion of studies scoring 0 and above according to coding method in this study. †Inclusion of moderate and strong studies according to EPHPPT qualitative summary score. łInclusion of studies scoring + or ++ according to GATE qualitative summary score for internal and external validity.

SSee table 2 for an overview of included studies.

CASP, Critical Appraisal Skills Programme; EPHPPT, Effective Public Health Practice Project tool; GATE, Graphical Appraisal Tool for Epidemiological Studies; NOS, Newcastle-Ottawa Scale; ROB, Cochrane Collaboration Risk of Bias tool.

standard for exposure and health outcome assessment based on sound subject matter knowledge, as proposed by Liverpool, improves validity of ratings and inter-rater reliability.

\section{Quality of component answers}

OATs employ multiple-choice answers, quantitative answers and categorical answers, with each approach presenting with advantages and disadvantages. The multiple-choice answers in the NOS limit subjectivity where answer categories apply; missing categories turned out to be a problem for some studies. Quantitative answers can increase precision (eg, response rate in EPHPPT) or be misleading. For example, the results of a study that adjusts for more than $80 \%$ of relevant confounders may nevertheless be highly confounded if one main confounder (eg, malnutrition in the case of diarrhoea) is missing. Categorical answers (eg, low, moderate, high in ROB) worked well in most cases but too few answer categories can be a problem (eg, CASP).

The meaning of the categories 'can't tell' and 'not reported' is also not unequivocal. For example, a strict interpretation of 'not reported' for blinding would be that researchers and study participants were not blinded (ie, coding of -1), and a less strict interpretation would be that owing to poor reporting we do not know whether the study was blinded (ie, coding of 0 ).

\section{Recommendations for the use of OATs in systematic reviews}

Our main meta-analysis yielded a pooled effect estimate that is more precise than those derived through sensitivity analyses and shows greater intervention effectiveness than more recently conducted systematic reviews. ${ }^{11-15}$ This finding strongly suggests that it is better to use any OAT than to ignore quality appraisal altogether. We felt that we had really understood a study after having applied two or three OATs, implying that none of the tested OATs covers all critical components. Therefore, we recommend (1) testing of a broader set of OATs on a more up-to-date systematic review of a public health intervention covering a wide range of epidemiological study designs and (2) research into the development of a reliable OAT with broad applicability across study designs.

What features should this gold standard OAT adhere to based on our experience? First, it is difficult to meet the needs of all study designs on a level playing field; instead, a set of companion

\section{What is already known on this subject}

Systematic reviews of public health interventions are a cornerstone of evidence-based decision-making but present with a range of methodological challenges, including which observational study designs to include and how to assess their quality.

- With more than 200 different tools available, a standard approach regarding quality appraisal of observational studies does not exist and the impact of the choice of tool on the results of systematic reviews is unknown.

\section{What this study adds}

- Our study shows large heterogeneity in the assessment of study quality between six widely used quality appraisal tools. Conducting meta-analysis for only moderate to high quality studies as identified by each tool yields differences in size and precision of the pooled effect estimate.

- Our study emphasises the importance of quality appraisal in systematic reviews, and the need to develop and test a gold standard tool that is applicable across a broad range of epidemiological study designs and addresses all major aspects of internal and external validity.

OATs comprising common components and study designspecific components could initially be developed for standard intervention and observational study designs and then be expanded to address more complex designs. Second, an overall summary score may lead to information loss and oversimplification. Instead, we propose a careful quantification of domains (rather than individual questions) in a weighted checklist, where results could be graphically illustrated using the traffic light scheme or quantified using our coding approach. Third, the gold standard OAT should address both internal and external validity with a clear distinction between these. Fourth, much more effort should be invested into the development and testing of appropriate and specific questions and answer categories; a manual can help with their correct interpretation. Finally, we believe that the subject-specific adaptation of selected questions makes quality appraisal more precise and reliable than a one size fits all OAT.

We encourage those interested in conducting systematic reviews including observational studies and in making public health more evidence based to take forward some of these ideas and recommendations for research.

Acknowledgements We would like to thank James R Miller for his support in developing the coding method. We are most grateful to Hajo Zeeb and the three anonymous reviewers for their insightful and constructive comments on a previous version of this article.

Contributors EAR had the original idea for this research; PV and EAR contributed actively to the design, analysis and interpretation, drafting the article and revising it critically. Both the authors read and approved the final manuscript.

Funding Eva Rehfuess gratefully acknowledges financial support from the Munich Center of Health Sciences.

Competing interests None.

Provenance and peer review Not commissioned; externally peer reviewed. 


\section{REFERENCES}

1. Brownson RC, Fielding JE, Maylahn CM. Evidence-based public health: a fundamental concept for public health practice. Annu Rev Public Health 2009;30:175-201.

2. Lavis J, Posada F, Haines A, et al. Use of research to inform public policy-making. Lancet 2004;363:1615-21.

3. Egan M, Petticrew M, Ogilvie D. New roads and human health: a systematic review. Am J Public Health 2003;93:1463-71.

4. Ogilvie D, Egan M, Hamilton V, et al. Systematic reviews of health effects of social interventions: 2. Best available evidence: how low should you go? J Epidemiol Community Health 2005;59:886-92.

5. Deeks JJ, Dinnes J, D'Amico R, et al. Evaluating non-randomised intervention studies. Health Technol Assess 2003; 7:iii-x, 1-173.

6. Katrak P, Bialocerkowski AE, Massy-Westropp N, et al. A systematic review of the content of critical appraisal tools. BMC Med Res Methodol 2004:4:22.

7. Sanderson S, Tatt ID, Higgins JP. Tools for assessing quality and susceptibility to bias in observational studies in epidemiology: a systematic review and annotated bibliography. Int J Epidemiol 2007;36:666-76.

8. ECDC. Evidence-Based Methodologies for Public Health: How to Assess the Best Available Evidence When Time is Limited and there is Lack of Sound Evidence. Stockholm: European Centre for Disease Prevention and Control, 2011.

9. Curtis V, Cairncross S. Effect of washing hands with soap on diarrhoea risk in the community: a systematic review. Lancet Infect Dis 2003:3:275e81.

10. Institute for Health Metrics and Evaluation. Global Burden of Disease Study. 2010. http://www.globalburden.org/ (accessed 23 Jul 2011).

11. Aiello $\mathbf{A E}$, Coulborn RM, Perez V, et al. Effect of hand hygiene on infectious disease risk in the community setting: a meta-analysis. Am J Public Health 2008;98:1372-81.

12. Ejemot RI, Ehiri JE, Meremikwu MM, et al. Hand washing for preventing diarrhoea. Cochrane Database Syst Rev 2008;(1):CD004265.

13. Fewtrell L, Colford JJ. Water, Sanitation and Hygiene: Interventions and Diarrhoea-A Systematic Review and Metaanalysis. Washington, DC: World Bank, 2004.

14. Fewtrell L, Kaufmann RB, Kay D, et al. Water, sanitation, and hygiene interventions to reduce diarrhoea in less developed countries: a systematic review and metaanalysis. Lancet Infect Dis 2005;5:42-52.

15. Waddington $\mathbf{H}$, Snilstveit $\mathrm{B}$, White $\mathrm{H}$, et al. Water, sanitation and hygiene interventions to combat childhood diarrhoea in developing countries. New Delhi: International Initiative for Impact Evaluation (3ie), 2009.

16. Cairncross S, Hunt $C$, Boisson $S$, et al. Water, sanitation and hygiene for the prevention of diarrhoea. Int J Epidemiol 2010;39(Suppl 1):i193-205.

17. Ciliska D, Fitzpatrick-Lewis D, Peirson L, et al. Effective Public Health Practice Project Tool. http://www.ephpp.ca/Tools.html (accessed 27 Jul 2011).

18. Critical Appraisal Skills Programme. CASP appraisal tools. Oxford: Public Health Resource Unit, 2006. http://www.sph.nhs.uk/sph-files/casp-appraisal-tools (accessed 23 Jul 2011)

19. Wells GA, Shea B, O'Connell D, et al. The Newcastle-Ottawa Scale (NOS) for assessing the quality of nonrandomised studies in meta-analyses. http://www.ohri. ca/programs/clinical epidemiology/oxford.asp (accessed 23 Jul 2011).

20. Dherani $\mathbf{M}$, Pope $\overline{\mathbf{D}}$, Mascarenhas $\mathbf{M}$, et al. Indoor air pollution from unprocessed solid fuel use and pneumonia risk in children aged under five years: a systematic review and meta-analysis. Bull World Health Organ 2008;86:390C-8C.
21. Jackson R, Ameratunga $\mathrm{S}$, Broad J, et al. The GATE frame: critical appraisal with pictures. Evid Based Med 2006;11:35-8.

22. Reeves B. Assessing Risk of Bias to Non-Randomised Studies (NRS) [For Systematic Reviews]. 2009. http://www.evidencebasedpublichealth.de/download/ ROB_tool_Reeves.pdf (accessed 23 Jul 2011).

23. Jüni $\mathbf{P}$, Altamn D, Egger M. Systematic reviews in health care: assessing the quality of controlled clinical trials. BMJ 2001;7:42-6.

24. Hussein Gasem M, Dolmans W, Keuter M, et al. Poor food hygiene and housing risk factors for typhoid fever in Semarang, Indonesia. Trop Med Int Health 2001;6:484-90.

25. Birmingham ME, Lee LA, Ntakibirora M, et al. A household survey of dysentery in Burundi: implications for the current pandemic in sub-Saharan Africa. Bull World Health Organ 1997;75:45-53.

26. Han A, Hlaing T. Prevention of diarrhoea and dysentery by hand washing. Trans $R$ Soc Trop Med Hyg 1989;83:128-31.

27. Hoque BA, Chakraborty J, Chowdhury JT, et al. Effects of environmental factors on child survival in Bangladesh: a case control study. Public Health 1999;113:57-64.

28. Khan MU. Interruption of shigellosis by hand washing. Trans $R$ Soc Trop Med Hyg 1982;76:164-8.

29. Khin MU, Myo K, Nyunt NW, et al. Risk factors for persistent diarrhoea and malnutrition in Burmese children. II: behaviour related to feeding and hand washing. $J$ Trop Pediatr 1994:40:44-6.

30. Peterson EA, Roberts L, Toole MJ, et al. The effect of soap distribution on diarrhoea: Nyamithuthu Refugee Camp. Int J Epidemiol 1998;27:520-4.

31. Shahid NS, Greenough WB 3rd, Samadi AR, et al. Hand washing with soap reduces diarrhoea and spread of bacterial pathogens in a Bangladesh village. J Diarrhoeal Dis Res 1996;14:85-9.

32. Sircar BK, Sengupta PG, Mondal SK, et al. Effect of handwashing on the incidence of diarrhoea in a Calcutta slum. J Diarrhoeal Dis Res 1987;5:112-14.

33. St Louis $\mathbf{M}$, Procter J, Helal A, et al. Epidemic cholera in West Africa: the role of food handling and high-risk foods. Am J Epidemiol 1990;131:719-28.

34. Velema JP, van Wijnen G, Bult P, et al. Typhoid fever in Ujung Pandang, Indonesia-high-risk groups and high-risk behaviours. Trop Med Int Health 1997:2:1088-94.

35. Wilson JM, Chandler GN, Muslihatun, et al. Hand-washing reduces diarrhoea episodes: a study in Lombok, Indonesia. Trans R Soc Trop Med Hyg 1991;85:819-21.

36. Yeager B, Lanata C, Lazo F, et al. Transmission factors and socio-economic status as determinats of diarrhoeal incidence in Lima, Peru. J Diarrhoeal Dis Res 1991:9:186-93.

37. Des Jarlais DC, Lyles C, Crepaz N. Improving the reporting quality of nonrandomized evaluations of behavioral and public health interventions: the TREND statement. Am J Public Health 2004;94:361-6.

38. Schulz KF, Altman DG, Moher D. CONSORT 2010 statement: updated guidelines for reporting parallel group randomised trials. J Clin Epidemiol 2010;63:834-40.

39. von Elm E, Altman DG, Egger M, et al. The Strengthening the Reporting of Observational Studies in Epidemiology (STROBE) statement: guidelines for reporting observational studies. J Clin Epidemiol 2008;61:344-9.

40. Cooper H. The Integrative Research Review: A Systematic Approach. Newbury Park, CA: Sage, 1984. 